\title{
The prototype of IoT Technological Infrastructure for Monitoring Vital Signs in Patients in an Emergency Department
}

\author{
Hernández-Hernández Guillermo Carlos, $\mathrm{Msc}^{1}$, Romero-Garavito Miguel Ángel, $\mathrm{Msc}^{2}$ y Rodríguez-Manrique \\ Jhonatan Andrés, $\mathrm{Msc}^{3}$ \\ ${ }^{1}$ Corporación Universitaria del Caribe CECAR, Guillermo.hernandez@cecar.edu.co. \\ ${ }^{2}$ Corporación Universitaria del Caribe CECAR, Colombia, Miguel.romero@cecar.edu.co. \\ ${ }^{3}$ Corporación Universitaria del Caribe CECAR, Colombia, jhoejo87@gmail.com.
}

\begin{abstract}
In Colombia, emergency services are characterized by the great congestion that occurs in institutions that provide this type of care to the community. The goal of this study was to perform a Prototype of IoT Technological Infrastructure for Monitoring Vital Signs in Patients in an Emergency Department. Methodologically, five stages were developed that consisted of characterizing the primary care process in the emergency department of a public institution, then the design of the prototype of technological infrastructure was carried out, followed by the construction of the prototype of technological infrastructure and finally the evaluation of the prototype of technological infrastructure. it is concluded that development of the project allowed to verify the feasibility of using current and innovative technologies such as IoT, in real environments, under conditions with scarce technological resources and with critical processes, such as those of a public health institution.
\end{abstract}

Keywords-- IoT, urgency, vital signs, monitoring, triage

Infrastructure.

\section{INTRODUCTION}

In Colombia, emergency services are characterized by the great congestion that occurs in institutions that provide this type of care to the community. The congestion situation corresponds to a problem that leads to poor care and lifethreatening risks for patients [1]. According to District statistics for the year 2012: occupation in hospitalization services of obstetrics and gynecology is 14.5\%; internal medicine $15.4 \%$; surgical $10.9 \%$; pediatrics $10.6 \%$; basic neonatal care $10.2 \%$; psychiatry $12.4 \%$; and emergency observation $13.7 \%$ in hospitals of the third level of care. In the second level, the hospitalization services that present an overquota are general medicine with $12.1 \%$; internal medicine with $11 \%$, and emergencies with $25.2 \%$ [2].

Another transcendental aspect that is part of the problem of emergencies in healthcare institutions has to do with the fact that most emergency care centers in the country suffer from appropriate technologies and mechanisms for adequate realtime supervision of patients who agree to request their services. These institutions do not have elements that allow them to monitor the vital signs of patients in waiting rooms and with this information, make the best decisions that allow them to improve the quality of health care for patients and thereby contribute to reducing the risks to their lives. In relation to this, new technologies currently play a fundamental role in supporting health processes in the institutions that provide these services [3]. The use of technology has become a promising solution for improving the service provided by institutions that have decided to include it as a service improvement strategy.

In relation to the above, it should be mentioned that the use of technologies in the context of health has led to the creation of a special implementation called e-Health [4], whose main purpose has been the development of technological tools for prevention, treatment, follow-up, and diagnosis in health in order to improve the efficiency of this service [5]. e-Health developments have been successfully applied to solve various health-related problems [6][7][8], due to the potential of these implementations to obtain information from patients and in general from health processes, becoming a support for decision making by responsible medical personnel in the organizations.

A concept that has been frequently used in e-Health implementations corresponds to the Internet of Things (IoT), which has been developing over the last few years impacting society in different areas and aspects of everyday life. Internet developments of things specifically in the health environment have involved the use of various types of devices, methodologies, and techniques that have led to the development of diverse and sophisticated solutions in this particular field [9][10]. The fusion of the Internet of things and multimedia technologies has penetrated the health field in order to provide life support and telemedicine [11].

In relation specifically to emergency or urgent services and Internet solutions of the things can be mentioned investigations that solve the problem of ubiquity, real-time data collection, among others, through the use of wired and/or wireless technologies to achieve the desired objectives. IoT technology provides value to emergency response operations in terms of effective cooperation, accurate situational awareness, and full visibility of resources [12][13]. 
In the present study proposes a prototype of technological infrastructure based on Internet of things for the vital signs monitoring in patients of an emergency service. This implementation searches for the permanent monitoring of the vital signs of patients waiting to be attended by the doctors of this unit, improving the decision-making of the medical personnel and diminishing the risks of the health of the users of the services of health.

\section{MATERIALS AND METHODS}

This study was carried out in the city of SincelejoDepartment of Sucre, in an institution that provides public health services. Initially, a phase of characterization of the institution's care process was carried out, the objective was to specify the process of first care for a patient requesting the emergency department, by identifying all the actors involved in the initial care process for patients in this dependency. In addition, the process of recording vital signs and the permissive limits of their risks for a patient accessing emergency departments was characterized. Likewise, the geographical areas dedicated to emergency care in the institution were determined and finally, the technological infrastructure installed in that institution was observed.

In order to develop this stage, two main sources of primary information were used as a reference, which were interviews conducted with all actors involved in the first care process for emergency patients (watchman, triage personnel, administrative officers and emergency physicians) and also by means of detailed observation of the process, following up on several patients who arrived at the institution requesting emergency medical attention. At the end of the characterization phase, the second stage of the design of the technological infrastructure was carried out, taking into account the existing infrastructure of the Institution. To this end, three procedures were basically executed, the first was to design a general prototype of technological infrastructure; later on, the construction of the software prototype was carried out; and finally, the construction of the electronic device for monitoring vital signs (DEMIOT). The construction of this device included as main materials: Arduino Uno Rev3 board, e-Health Sensor v2.0 board, WIFI module ESP8266, SpO2 sensor and the e-Health blood pressure sensor version 2 .

After the design stage of the technological infrastructure, the prototyping stage was completed. This stage was divided into two main activities, the first related to the construction of the software and the second to the construction of the electronic monitoring device of IoT. Taking into account that the developed corresponds to a functional prototype and not to a final system in production, it was decided to choose The methodology for developing XP programming software was chosen as the guiding guide for the process. It is important to mention that this methodology was adapted to serve as a way forward in the process of building the prototype hardware. Specifically, the adaptation carried out addressed the entire construction process of the electronic monitoring device called DEMIOT. Finally, the stage of the evaluation of the prototype was carried out, where the functionality of the prototype was evaluated and the feasibility of using it as a decision-making mechanism within an emergency department. To do this, it was necessary to validate the prototype developed and evaluate its operation.

\section{RESULTS AND DISCUSSION}

Once the pertinent instruments for the collection of primary information from the personnel of the different units related to the institution's emergency department were applied, it was established that the standard process of first emergency care was consistent with what is illustrated in figure 1.

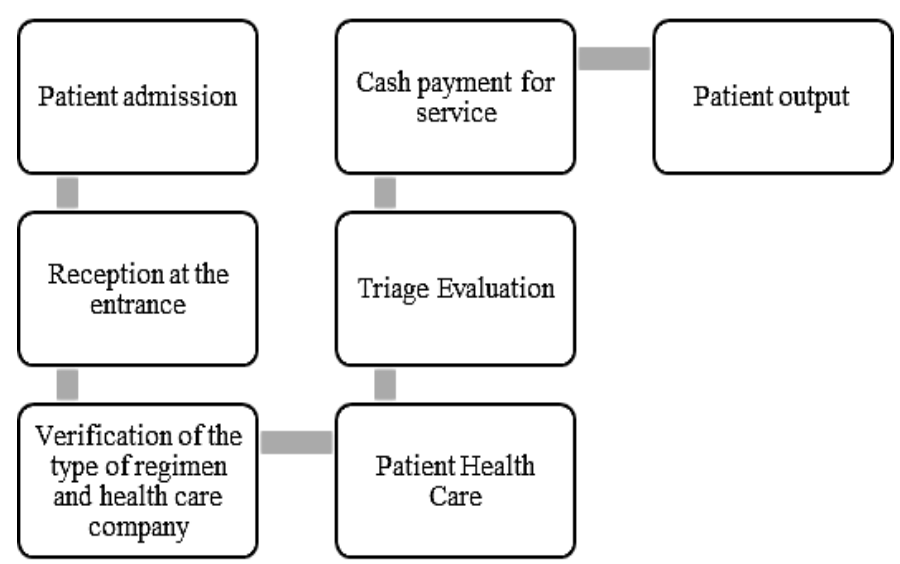

Fig. 1 General process of patient care in the emergency department.

Regarding the technological infrastructure, the administrative officials stated that they had the characteristics described in Table I.

TABLE I

ASPECTS RELATED TO TECHNOLOGICAL INFRASTRUCTURE IN THE INSTITUTION UNDERSTUDY.

\begin{tabular}{|l|c|}
\hline \multicolumn{1}{|c|}{ Items } & Found \\
\hline $\begin{array}{l}\text { Number of computer equipment } \\
\text { in the emergency department }\end{array}$ & 6 \\
\hline Number of computers in triage & 1 \\
\hline $\begin{array}{l}\text { Number of computers at the } \\
\text { entrance }\end{array}$ & \multicolumn{1}{c|}{ Yes (Private use) } \\
\hline Internet Access & Yes (does not work correctly) \\
\hline WIFI & 0 \\
\hline Internal data network & 0 \\
\hline Data Servers & \multicolumn{1}{c|}{0} \\
\hline $\begin{array}{l}\text { Medical Devices with Internet } \\
\text { connection support }\end{array}$ & $\begin{array}{l}\text { Medical Records (Not used in the } \\
\text { emergency department). }\end{array}$ \\
\hline Software & - Invoicing \\
\hline
\end{tabular}


As for the triage unit, the staff interviewed stated that the emergency service in the institution begins when the patient arrives at the goal and is received by the security guard who takes your personal information (Name, last name). The caretaker guides the patient through the steps to be taken in order to be attended (if it is to make copies or to have a stamp placed on the patient's billing, or other information). Once their check-in procedures have been resolved, the patient is gets triaged to an assistant and the head nurse who evaluates the patients and takes the vital signs information from the patients. The vital signs that are usually recognized in patients are pulse or heart rate, breathing rate, blood pressure and temperature. In this sense, the triage staff explained that they have a team that allows obtaining all the information related to vital signs and that their results are recorded on a sheet of paper called the triage format, this works as devices or instrument to measure these indicators in the institution. Once the patient has been evaluated in triage depending on the classification, they are immediately transferred to medical attention or taken to the waiting rooms to be called for treatment. It is emphasized that patients are not given vital signs again after being treated in triage and during the waiting process for treatment. In summary, figure 2 illustrates the process of first care for patients entering the institution's emergency department.

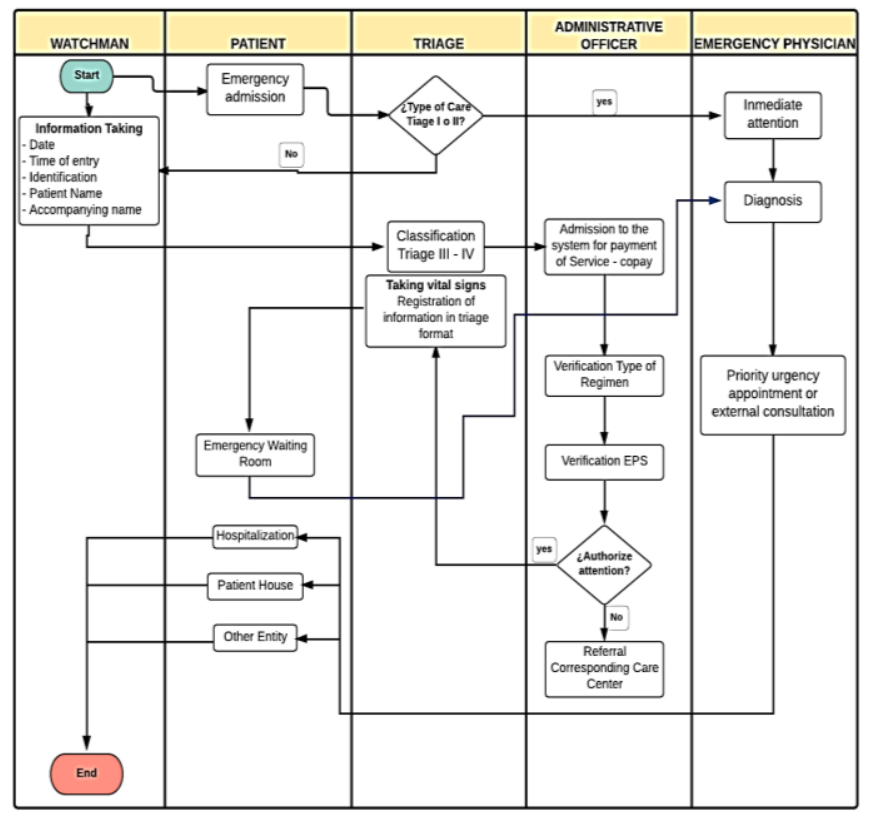

Fig. 1 General process of patient care in the emergency department.

The stage corresponding to the design of the technological infrastructure proposed a technological architecture that, in addition to the monitoring of vital signs, would allow automating the first attention process of an emergency service. The infrastructure designed took into account the processes previously erected, the devices and equipment used in the current emergency processes of the institution under study. The overall design of the technological infrastructure prototype responded to a set of functional requirements raised during the characterization phase of the project. These were divided into two types, software-associated and Hardware associated requirements, table II below shows the main requirements of each type.

TABLE II

MAIN SOFTWARE AND HARDWARE REQUIREMENTS

\begin{tabular}{|c|c|}
\hline Software Requirements & Hardware Requirements \\
\hline $\begin{array}{l}\text {-The system will allow the } \\
\text { registration of patients with } \\
\text { incomplete mandatory data. }\end{array}$ & $\begin{array}{l}\text {-The system will allow the } \\
\text { monitoring of a vital sign of patients } \\
\text { waiting for medical attention in the } \\
\text { emergency department. }\end{array}$ \\
\hline
\end{tabular}

-The system will allow authorized users (triage) to record vital signs of patients awaiting medical attention in the emergency department.

-The system will use a unique identifier per patient that will allow identification of the patient during his or her stay in the emergency department.

-The system will allow the sending of information of a vital sign taken from the patient, for subsequent registration and monitoring.

-The system will be portable and will allow free movement and manipulation of the patient under permanent monitoring.

-The system will allow authorized users (triage) to monitor vital signs of patients awaiting the attention of physicians in the emergency department.

-The system will allow patients to receive vital signs information from an electronic device implemented for this purpose.

-The system will allow alerts to be generated when a variation in the parameters of a patient's vital signs is received outside the patient's acceptance ranges.

These requirements led us to propose the prototype of technological infrastructure, which response to a client-server architecture where the different direct actors of the first attention to the emergency patient intervene carrying out the activities that are established within the specific functions of their role and that have been modeled in the prototype. Once the patient arrives at the emergency room, their personal data and that of their companion are recorded, then the vital signs are measured and according to their classification in triage, medical attention is carried out or sent to the waiting room. Users who are sent to the waiting room are placed on a device that allows them to monitor their vital signs. For this prototype, the heart rate or pulse rate and oxygen concentration were monitored automatically. Blood pressure is measured semi-automatically in the device, i. e. the patient or nurse must be involved in the process. The information from these measurements is sent to the server in charge of reporting 
the monitored values of vital signs to the medical personnel in charge. The overall structure of the technological infrastructure prototype is shown in Figure 2.

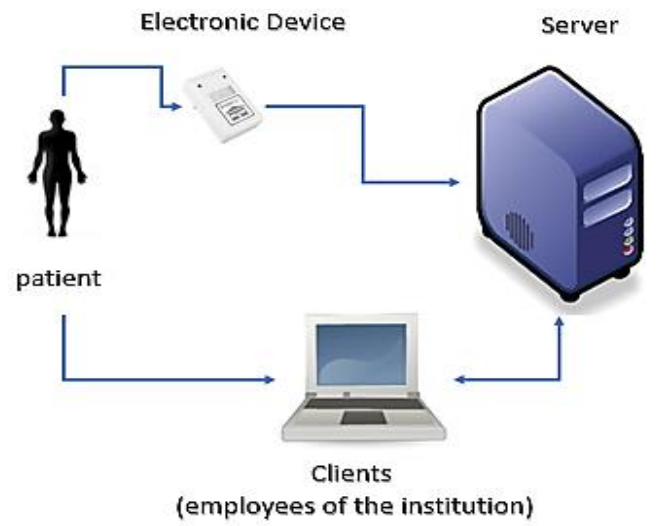

Fig. 2 General diagram of the technological infrastructure prototype

The detailed design of the technological infrastructure prototype includes three main elements. Initially the clients, who are employees of the institution involved in the process of first care of patients who access an emergency department, which, using web browsers, record and consult information about the users of this medical service. It should be clarified that the system's client computers are of varied specifications, the institution's desktop computers, laptops and even mobile devices that were used by staff members with diverse roles that are part of the prototype technological infrastructure. A second fundamental element corresponds to the System Server, which receives requests from clients, communicates with the database management system and returns information to clients. It has an x86 architecture processor under a Linux Ubuntu 14.04 operating system. The third fundamental element corresponds to the electronic monitoring device (DEMIoT), which keeps taking information from the patient waiting to be attended by the emergency physicians of the institution.

The detailed operation process of the technological infrastructure prototype begins when the shift watchman, once he has started his working day, accesses the system with his username and password. Once you have performed this operation, you are ready to receive patients. The patient who approaches the institution requesting emergency care is received by the warden who receives it and verifies the patient's condition, if he or she does not require immediate attention, the patient is registered in the system by entering the corresponding information, as well as that of his or her companion (if accompanied). The patient is then welcomed in the triage section where he or she is cared for by the nurse-inchief or auxiliary nurses who are responsible for evaluating and classifying him or her according to the categories of triage. In this procedure, the triage staff takes patients' vital signs in a traditional way with the instruments they usually use for this task and records them in the application. It is worth mentioning that the person in charge in the triage of the vital signs information registration has had to enter the system previously with his username and password. Once the patient's vital signs have been recorded and it has been determined that it corresponds to a patient who is going to go to the waiting room to wait for treatment by emergency physicians, an electronic monitoring device is assigned to the patient. The triage person enters the application and assigns the electronic monitoring device (DEMIoT) to the patient's identification number, and then proceeds to place it on a part of the body that does not affect the patient's medical condition and does not discomfort him/her during the waiting period. After the DEMIoT is assigned, it is switched on and the patient is sent to the waiting room while waiting for care. It is important to note that considering the general hardware requirements mentioned above, when mention is made of the portability of the electronic monitoring device, it was considered for operation using Wireless Fidelity (WIFI) technology.

While the patient is in the waiting room, the triage staff has a monitor for constant patient monitoring. And to watch for variations and a possible presence of alert at the moment when a variation occurs outside the permitted limits of the monitored vital sign. Once the patient is requested for care by the emergency physician, the triage staff are responsible for removing the DEMIoT. Patient care ends with the patient's discharge order from the emergency physician, which is recorded by the institution's custodian in order to close the patient care process.

Once the technological infrastructure design stage was completed, the prototype of technological infrastructure was developed as shown in figure 3 .

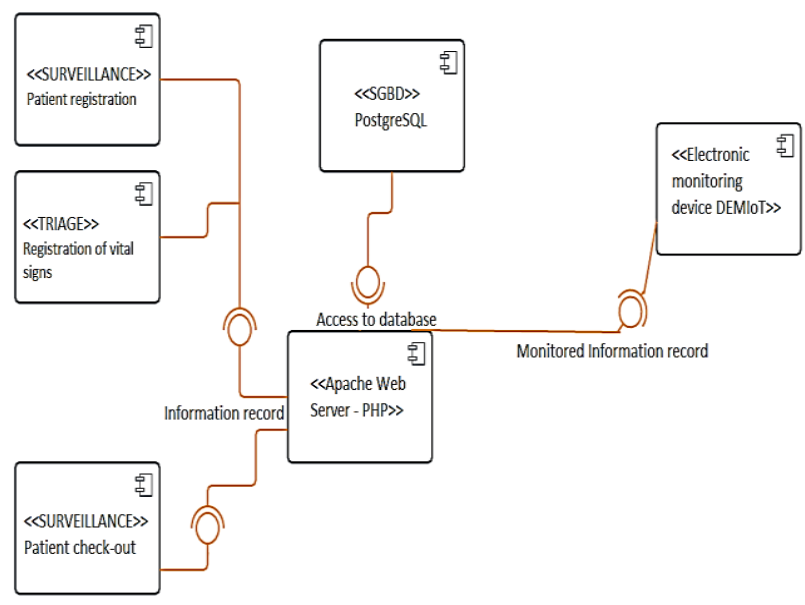

Fig. 3 General diagram of the technological infrastructure prototype

The sub-stage corresponding to the construction of the software prototype was directed taking into account the 
existing infrastructure in the public institution since the evaluation of the built prototype would be carried out in this institution. As mentioned above, the software development methodology that guides the project corresponds to the XP methodology, taking into account the concepts associated with it, defined the stages that would guide the construction of software, which were the Exploration Phase, Planning Phase, Iteration Phase and the Production Phase.

The application of the XP methodology began by defining the roles associated with the project. It is important to note that the project development team consisted of three people, a developer, testing manager, follow-up manager and Coach; a second person assumed the role of Consultant; and a third person who assumed the Big Boss activities. For the documentation of the prototype requirements obtained, the user histories were used and the delivery schedules were also established, as well as a specification of the user histories to be developed for each iteration. An estimate was also made of the effort required for each of them and the construction priority. The implementation of the user stories allowed obtaining information related to the approximate measurement of effort.

Regarding the definition of acceptance tests, acceptance tests were carried out in each of the iterations and at these stages, the client proposed the corresponding adjustments to arrive at the acceptance of this requirement. Four iterations were carried out for the construction of the software associated to the technological infrastructure prototype, and each one of them used the specification of the tasks that by means of the CRC cards and engineering tasks. In this same order, the substage corresponding to the construction of the monitoring device, presented as an innovative aspect the adaptation of the XP software development methodology, in order to be used as part of the hardware construction process. Although it is true that this element is not a software product and that a hardware development methodology would be much more appropriate, an adjustment was made to the XP methodology so that it could be applied and allowed to guide methodologically the construction of this device. In iteration 4 of the project, the second cycle of XP methodology oriented exclusively to the development of the DEMIoT electronic monitoring device was demonstrated.

The Exploration Phase associated with the construction of the device made it possible to evaluate various technologies with which DEMIOT could be implemented. It is important to note that for the purpose of the comparison it was taken into account that the vital sign to be measured would correspond to the pulse or heart rate; the solution should consider wireless communication via Bluetooth or Wifi; the possibility of constructing a portable device and adjustable to the emergency patient.
In order to compare the alternatives, the possible construction of the devices with each of the technologies was investigated. It should be noted that the cost values presented in Table III relate only to the solution motherboard and do not include additional modules, sensors or any other devices required for the implementation of the device.

TABLE III

COMPARISON OF TECHNOLOGIES FOR CONSTRUCTION OF THE ELECTRONIC MONITORING DEVICE

\begin{tabular}{|c|c|c|c|c|}
\hline Items & Nodemcu & Arduin & $\begin{array}{c}\text { Raspberry } \\
\mathbf{P i}\end{array}$ & $\begin{array}{c}\text { Pic } \\
\text { Microcontroller }\end{array}$ \\
\hline $\begin{array}{l}\text { The } \\
\text { existence } \\
\text { of the } \\
\text { product in } \\
\text { health } \\
\text { sensors }\end{array}$ & $\begin{array}{l}\text { NodeMC } \\
\text { U ESP- } \\
\text { 12E (Not } \\
\text { a health- } \\
\text { specific } \\
\text { solution) }\end{array}$ & $\begin{array}{c}\text { Libelium } \\
\text { e-Health } \\
\text { v2.0 } \\
\text { Platform } \\
\text { My } \\
\text { Signals } \\
\text { Platform }\end{array}$ & $\begin{array}{c}\text { Raspberry } \\
\text { Pi Model B } \\
\text { (Not a } \\
\text { health- } \\
\text { specific } \\
\text { solution) }\end{array}$ & $\begin{array}{c}\text { PIC16F628A } \\
\text { (Not a health- } \\
\text { specific } \\
\text { solution) }\end{array}$ \\
\hline Cost & EUR 9 & $\begin{array}{c}\text { e-health } \\
\text { EUR } \\
52.50 \\
\\
\text { My } \\
\text { Signals } \\
\text { EUR 379 }\end{array}$ & EUR 49.95 & EUR 12.99 \\
\hline $\begin{array}{c}\text { Free } \\
\text { Solution }\end{array}$ & Yes & Yes & $\begin{array}{c}\text { Proprietary } \\
\text { product } \\
\text { with } \\
\text { registered } \\
\text { ownership, } \\
\text { but for free } \\
\text { use }\end{array}$ & Not \\
\hline $\begin{array}{c}\text { Documenta } \\
\text { tion }\end{array}$ & Medium & High & High & Medium \\
\hline Ease of use & Medium & High & High & Medium-High \\
\hline $\begin{array}{c}\text { Ease of } \\
\text { acquisition } \\
\text { in the } \\
\text { market }\end{array}$ & High & $\begin{array}{l}\text { Medium- } \\
\text { High }\end{array}$ & $\begin{array}{l}\text { Medium- } \\
\text { High }\end{array}$ & Medium-High \\
\hline $\begin{array}{l}\text { Adaptabilit } \\
\text { y to project } \\
\text { requiremen } \\
\text { ts }\end{array}$ & Medium & High & $\begin{array}{l}\text { Medium- } \\
\text { High }\end{array}$ & Low \\
\hline
\end{tabular}

To evaluate the previously compared technologies, a weighting was assigned that depended directly on the conditions and requirements of the project. For the evaluation of the cost aspect, it was taken as a basis that the least expensive solution had a valuation of 10 and the others were proportional according to their respective costs. The assessment of each aspect of each of the technologies was established with values from 1 to 10 and is illustrated in Table IV. 
TABLE IV

EVALUATION OF TECHNOLOGIES FOR THE CONSTRUCTION OF THE ELECTRONIC MONITORING DEVICE.

\begin{tabular}{|l|c|c|c|c|c|}
\hline \multicolumn{1}{|c|}{ Aspect } & $\%$ & Nodemcu & $\begin{array}{c}\text { Arduino } \\
\text { e-Health }\end{array}$ & $\begin{array}{c}\text { Arduino } \\
\text { My- } \\
\text { Signals }\end{array}$ & $\begin{array}{c}\text { Raspberry } \\
\text { Pi }\end{array}$ \\
\hline $\begin{array}{l}\text { Existence of } \\
\text { product in health } \\
\text { sensors }\end{array}$ & 15 & 1 & 10 & 10 & 1 \\
\hline Cost & 10 & 10 & 5 & 2 & 5 \\
\hline Free Solution & 20 & 10 & 10 & 10 & 4 \\
\hline Documentation & 10 & 5 & 10 & 10 & 10 \\
\hline Ease of use & 15 & 5 & 10 & 10 & 10 \\
\hline $\begin{array}{l}\text { Ease of acquisition } \\
\text { in the market }\end{array}$ & 10 & 10 & 7 & 7 & 10 \\
\hline $\begin{array}{l}\text { Adaptability to } \\
\text { project } \\
\text { requirements }\end{array}$ & 20 & 5 & 10 & 10 & 8 \\
\hline \multicolumn{1}{|c|}{ Totals } & 100 & 6,4 & 9,2 & 8,9 & 6,55 \\
\hline
\end{tabular}

Taking into account the previous results, we proceeded to plan the construction of the prototype taking into account the technology with better evaluation Arduino e-Health. The construction of the DEMIoT guided by the adaptation of the XP methodology was developed in three iterations, and in each one of the engineering tasks related to the integration with the software and the pertinent adjustments to it were carried out, according to the functional behavior of the DEMIoT device. The architecture of the third version of DeMIoT is illustrated in Figure 4.



Fig. 4 Third version of the DEMIoT Architecture.

The final version of DEMIoT included the components Arduino e-Health, Arduino Uno, Arduino Nano, and a Wifi module. In addition, a Bakelite plate was built to interconnect the different components and make the device more compact. A nine-volt rechargeable battery and the corresponding sensor for measuring heart rate and oxygen concentration in the blood were added to these components. The monitoring of vital signs corresponds to the most important functionality of the prototype, as it involves constantly receiving information on vital signs of patients who are waiting to be seen by emergency physicians. The interface in Figure 5 allowed this activity to be carried out.



Fig. 5 User interface for monitoring vital signs of the built prototype.

Finally, the evaluation phase of the technological infrastructure prototype was oriented to the process of deploying the technological infrastructure, as well as evaluating its operation and making a record of its operation and performance. It is important to point out that the software tests were carried out during the development of the project, using the XP methodology and the software and hardware tests carried out in the previous stage. This stage included the deployment sub-stages of the technological infrastructure prototype; validation of the DEMIoT electronic monitoring device, and evaluation of the prototype developed.

For the deployment, a set of elements necessary for the operation of the prototype technological infrastructure designed was incorporated into the institution's infrastructure. The first of these corresponded to a Wifi router that was configured for the performance test. Another element necessary for the operation of the technological infrastructure prototype corresponds to a test server that was connected to the institution's switch and for which a fixed IP address of the internal network was designated to perform the test. The diagram of the technological infrastructure with the insertion of the new elements is shown in figure 6 .

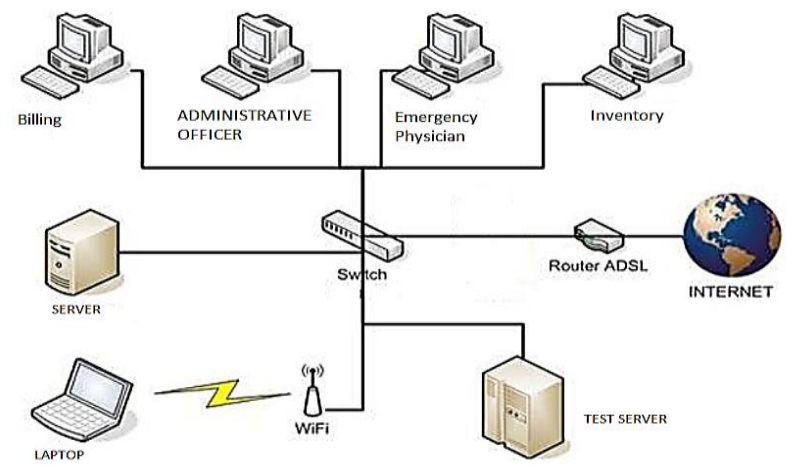

Fig. 6 Infrastructure of the public institution with prototype elements.

$1^{\text {th }}$ LACCEI International Multi-Conference for Engineering, Education, and Technology: "Innovation in Education and Inclusion", 19-21 July 2018, Lima, United States. 
Once the required elements in the institution's communications infrastructure were in place, the necessary installations and configurations were carried out, taking into account the infrastructure designed.

\section{CONCLUSIONES}

In the project, several trials were carried out with patients from the trial institution and non-patients in less critical conditions than in the emergency department. In relation to the methodology applied and the results obtained, it is concluded that the development of the project allowed to verify the feasibility of using current and innovative technologies such as IoT, in real environments, under conditions with scarce technological resources and with critical processes, such as those of a public health institution. In addition, the characterization of the first emergency care process made it possible to analyze the medical scope of this service and recognize the limited technological resources available to institutions for these tasks, in addition to the needs of professionals and users of emergency services with regard to the use of new technologies that improve their working procedures, leading to the reduction of human errors and improving the processes of this type of service. On the other hand, the infrastructure designed in this project becomes a contribution to existing solutions in the Internet of things and telemedicine. Finally, the application of this development in a real environment in order to observe and evaluate its functioning in an institution that provides health services becomes an important input when considering the implementation of this type of technologies at the macro level in an organization that provides this type of services.

\section{REFERENCES}

[1] Bravo, J., Flórez, D., \& Salazar, E. (2008). Servicios de Urgencias en Colombia, el caso Medellín. Medellín: Universidad CES.

[2] MALAVER, C. (24 de mayo de 2013). La falta de camas hace colapsar salas de urgencia en red hospitalaria. EL TIEMPO. Disponible en: http://www.eltiempo.com/archivo/documento/CMS-12822365.

[3] Sun-Yeob Kim1, Hyoung-Keun Park, Myoung-Kwan Oh, "Development of u-Healthcare System Based on Ubiquitous Networks", International Journal of Multimedia and Ubiquitous Engineering, Vol.9, No.1, pp.379390, 2014.

[4] Bazzani, Conzon, Scalera, Spirito, "Enabling the IoT paradigm in ehealth solutions through the VIRTUS middleware", IEEE 11th International Conference on Trust, Security, and Privacy in Computing and Communications, pp. 1954-1959, 2012.

[5] Swiatek, Rucinski, "IoT as a Service System for eHealth", IEEE HEALTHCOM - The 1st International Workshop on Service Science for e-Health, pp. 81-84, 2013.

[6] Abinaya, Raja Sekar, "HealthCare Information System for Emergency Medical Service based on Ubiquitous Data Accessing - Internet of Things", Special Issue on IEEE Sponsored International Conference on Intelligent Systems and Control (ISCO’15), Vol. 2, pp 163-166, 2014.

[7] Broom, Tovey, "The role of the Internet in cancer patients' engagement with complementary and alternative treatments healths", health: An Interdisciplinary Journal for the Social Study of Health, Illness and Medicine, Vol 12(2), pp. 139-155, 2008.
[8] Zhu, Diethe, Camplani, Tao, Burrows, Twomey, et al., "Bridging eHealth and the Internet of Things: The SPHERE Project", IEEE Computer Society, pp. 39-46, 2015.

[9] Jara, A., Zamora, M., \& Skarmeta, A., "An Internet of things-based personal device for diabetes therapy management in ambient assisted living (AAL)", Personal and Ubiquitous Computing, pp. 431-440, 2011.

[10] Gachet, De Buenaga, Aparicio, Padrón, "Integrating Internet of Things and Cloud Computing for Health Services Provisioning. The Virtual Cloud Carer Project", Sixth International Conference on Innovative Mobile and Internet Services in Ubiquitous Computing, pp. 918-921, 2012.

[11] Zhang, X., \& Zhang, N., "An open, secure and flexible platform based on the internet of things and cloud computing for ambient aiding living and telemedicine", International Conference on Computer and Management, 2011.

[12] Xu, B., Xu, L., Cai, H., Xie, C., Hu, J., \& Bu, F., "Ubiquitous Data Accessing Method in IoT-Based Information System for Emergency Medical Services", IEEE Transactions on industrial informatics, Vol. 10, No. 2, pp. 1578-1586, 2014.

[13] Yang, Yang, Plotnick, "How the Internet of things technology enhances emergency response operations", Technological Forecasting and Social Change, pp. 1-14, 2012. 